\title{
Integration and Reliability of Active Fiber Composite (AFC) Sensors/Actuators in Carbon/Epoxy Laminates
}

\author{
Mark M. Melnykowycz ${ }^{* a}$, Alberto Bellolib, Paolo Ermanni ${ }^{\mathrm{b}}$, Michel Barbezat ${ }^{\mathrm{a}}$ \\ ${ }^{a}$ EMPA, Swiss Federal Laboratories for Materials Testing and Research, Ueberlandstr. 129, 8600 \\ Duebendorf, Switzerland \\ ${ }^{\mathrm{b}}$ ETH Zurich, Centre of Structure Technologies, Leonhardstrasse 27, 8092 Zurich, Switzerland
}

\begin{abstract}
In the current study Active Fiber Composites (AFC) utilizing Lead-Zirconate-Titanate (PZT) fibers with Kapton ${ }^{\circledR}$ screen printed interdigitated electrodes (IDE) were integrated into carbon fiber reinforced plastic (CFRP) laminates to investigate integration issues associated with smart structures and host laminate integrity. To aid in this goal surrogate or "dummy" AFC (DAFC) using a composite core and Kapton ${ }^{\circledR}$ outer layers (to match the longitudinal mechanical and interface properties of the AFC) were employed. These DAFC were used in place of real AFC to expedite test specimen manufacture and evaluation. This allowed efficient investigation of the impact of an integrated AFC-like inclusion on laminate mechanical integrity. Laminates with integrated AFC were additionally tested with signal monitoring to assess AFC health during the test. Investigation into laminate failure was accomplished via a finite element model of the system which was created in ANSYS to investigate failure in the composite plies. Tsai-Wu failure criterion was calculated to investigate laminate failure characteristics. Integration of AFC into CFRP laminates degraded laminate strength by $13.3 \%$ using insertion integration and $7.8 \%$ using the interlacing integration technique. The finite element model showed that interlacing integration enabled distribution of critical forces over the entire laminate while insertion integration led to critical forces concentrating over the integration region.
\end{abstract}

Keywords: Active Fiber Composites, Integration, Failure, Sensor

\section{INTRODUCTION}

Due to excellent electrical-mechanical coupling piezoelectric materials based on Lead-Zirconate-Titanate (PZT) are good candidates for use in adaptive material systems. Traditional PZT devices are composed of a PZT wafer with electrodes on the top and bottom as in the Rainbow [1] and Thunder [2] actuators. However, these wafer based devices suffer from low damage tolerance and actuation performance. Improvement on these limitations was found in the work of Bent and Hagood [3] at the Massachusetts Institute of Technology in the original development of the Active Fiber Composite (AFC) actuator system. AFC are composed of PZT fibers embedded in an epoxy matrix and sandwiched between two interdigitated electrodes (IDE). The AFC design surpasses the traditional PZT wafer in many areas. Due to their composite design AFC are more flexible than traditional PZT wafers and they are polarized along the fiber length, as opposed to polarization through the wafer thickness, and thereby utilize the piezoelectric properties in the $\mathrm{d}_{33}$ direction, offering greater performance than wafers, which use the $\mathrm{d}_{31}$ component.

In the realm of smart materials design AFC are interesting because they can be designed for specific sensor and actuator applications. Many applications can be imagined for AFC such as health monitoring, actuation, and vibration damping. However, few studies have addressed the reliability and performance parameters of AFC or PZT wafer devices as a component in an adaptable structure. Many studies have addressed the ferroelastic and ferroelectric properties of bulk PZT $[4,5,6,7]$ which lends insight into the physical principles governing AFC. From the practical side, AFC characterization work has been performed for actuation performance and the specific application of angle of twist actuation of a Boeing active material rotor [8]. Less work however, has targeted the synergy between the active element

* Corresponding author. Tel.: +41-44-823-4307; fax: +41-44-823-4496. e-mail: mark.melnykowycz@empa.ch

Smart Structures and Materials 2006: Active Materials: Behavior and Mechanics, edited by William D. Armstrong, Proc. of SPIE Vol. 6170, 61701J, (2006) · 0277-786X/06/\$15 - doi: 10.1117/12.658639 
and the surrounding host structure. Use of AFC in a smart or adaptable materials application implies the integration of the devices into a host material. In many cases the host material is a laminar composite such as GFRP or CFRP. A number of applications are based on laminar composites and are using smart material principles such as tennis rackets [9], adaptive skis [10], and rotor blades [11,12]. To date, most research dealing with piezoelectric sensor/actuator integration has revolved around PZT wafer integration, with less research directed towards AFC-type devices. Therefore, investigating integration issues of AFC into composite laminates is relevant for many sectors.

\subsection{Integration}

Integration may be defined as any technique used to incorporate a piezoelectric device into a structure or material. In a laminate composite the two most basic integration techniques include "insertion" and "cutout insertion." Insertion entails simply placing the device between the laminate layers, essentially sandwiching it in place. Cutout insertion involves removing laminate material, and then placing the device into the void so that the device becomes continuous with the surrounding structure or material. Combining the two techniques leads to "interlacing." The interlacing technique involves moving the cutout ply away from the device. Crawley and Luis [13] investigated the integration of PZT wafer actuators into woven composites using analytical and experimental methods. In addition, they laid down design criteria for PZT actuators such as a high modulus of elasticity to allow stress transfer with the surrounding laminate. In static tension, the embedding of PZT devices were found to have little effect on the elastic modulus, but a $20 \%$ decrease in the ultimate tensile strength of glass/epoxy laminates embedded with PZT devices was observed.

Vizzini et al. [14,15] studied the effects of layering technique on the stress transfer between embedded PZT devices and unidirectional composite layers. In a first study [14] an optimal interlacing design was proposed using finite element techniques. The second study [15] tested the design by placing glass slides in uni-directional (UD) carbon/epoxy laminates and performing tensile testing. Paget et al. [16] performed tensile testing of carbon/epoxy laminates with inserted PZT wafers and only saw a $4.5 \%$ difference in ultimate strength, and a 3\% difference in Young's Modulus between the laminate with integrated PZT wafers and the reference laminate. In addition, multiple studies $[17,18,19,20]$ suggest that with quasi-isotropic carbon/epoxy composite lay ups the integration technique does not greatly affect the ultimate laminate strength. Mall et al. [19] reported no difference between the ultimate stress properties of composite laminates without integrated devices and those that employed the insertion and cutout integration techniques.

\subsection{Objectives}

Past work on AFC integration in woven GFRP laminates [21] has shown a pronounced affect on laminate integrity. This work seeks to build upon post findings concerning GFRP integration and investigate the performance of AFC integrated into CFRP laminates, which offers an integration environment much stiffer than that of the AFC. In the previous work dummy AFC (DAFC) were produced in order to optimize testing procedures, reduce manufacturing time and to predict the effect AFC integration would have on the integrity of the laminates. In order to simulate the AFC, DAFC were manufactured using Kapton ${ }^{\circledR}$ film and UD glass pre-preg plies to simulate the bonding characteristics with the laminate structure. The current work investigates AFC integration in CFRP laminates via experimental and numerical methods, considering the integration technique employed as well as the placement of the device in different ply positions through the thickness of the laminates. ANSYS was used to implement a numerical model with failure criterion to aid in understand how the laminates failed in relation to the integrated DAFC and AFC.

\section{MATERIALS AND METHODES}

\subsection{Materials}

CFRP laminates were manufactured using ELITREX pre-impregnated (pre-preg) CFRP composite plies (EHKF 420UD24K-40) supplied by Stesalit (www.stesalit.com). AFC were produced using $250 \mu \mathrm{m}$ diameter PZT-5A fibers produced by Smart Material Corp., Osprey Fl, USA (www.smart-material.com). The fibers were laminated with an 
epoxy system (Aradur 2954 hardener with Araldite LY564 resin) supplied by Vantico AG, Basel, Switzerland (www.vantico.com) and sandwiched between two sheets of $25 \mu \mathrm{m}$ thick Dupont Polyimide Kapton ${ }^{\circledR} 100 \mathrm{HN}$ film (www.dupont.com) with screen-printed IDE. Electrical connections for the AFC were made using $250 \mu \mathrm{m}$ POLYSOL180 wires supplied by Elektro-Feindraht AG. DAFC were fabricated using Stesatape EP121-GR101/228-30 UD glass pre-preg plies from Stesalit (www.stesalit.com) sandwiched between two sheets of $25 \mu \mathrm{m}$ thick $100 \mathrm{HN}$ polyimide Kapton ${ }^{B}$ film.

\subsection{Laminate specimen preparation}

CFRP laminates composed of 12 plies of UD pre-preg were manufactured with a [0;90 $]_{3 S}$ configuration. CFRP plies were cut to exact dimensions using a computer controlled Zünd M-1600 cutter. This allowed precise dimensioning to the cut out area for integration of DAFC and AFC, and reduced fiber misalignment that may have resulted from hand cutting of the integration area. Ply arrangement was performed by hand on an aluminum tooling plate. In addition, an aluminum frame was used to contain the plies on the tooling plate. The CFRP laminates were processed in an autoclave under 4 bar of pressure and cured at $120^{\circ} \mathrm{C}$ for 90 minutes. After processing the tensile specimens were cut from the composite plate with geometry and dimensions as shown in Fig. 2.1.

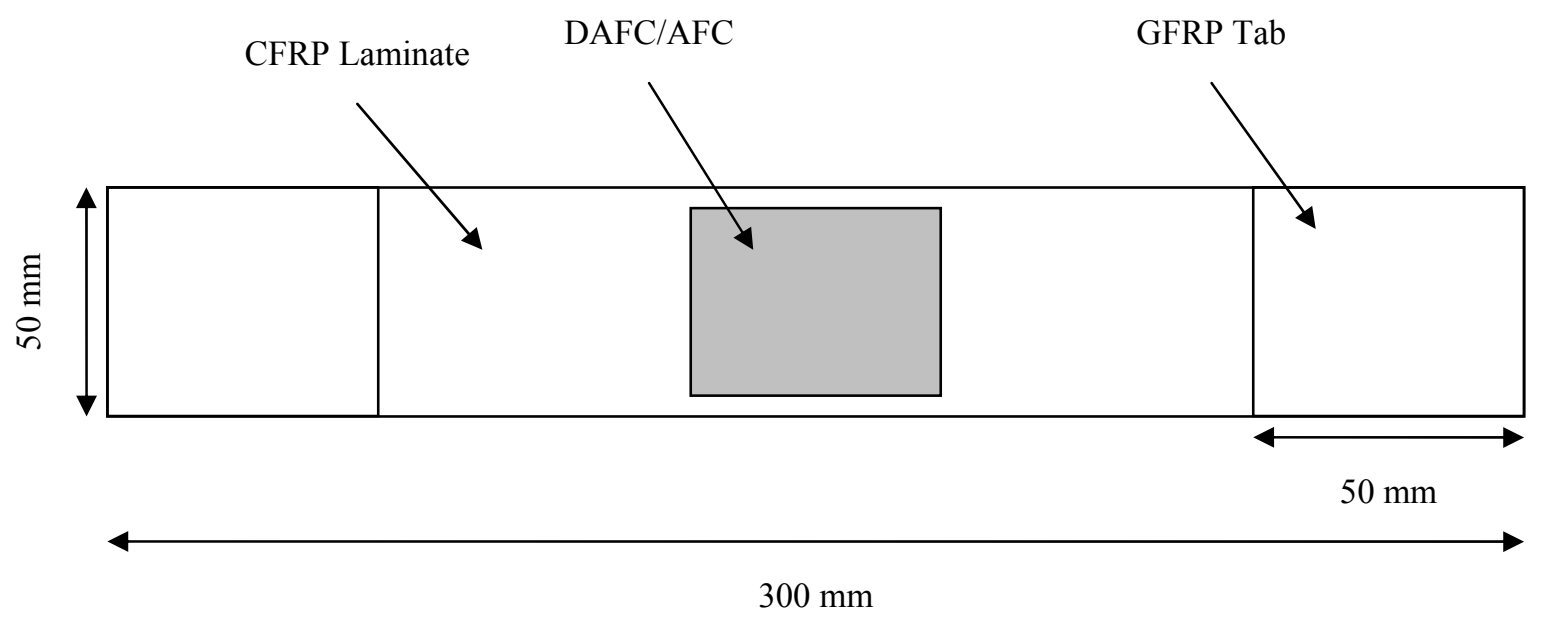

Figure 2.1 Tensile test specimen depicting the area of integration for the AFC/DAFC elements.

\subsection{Integration of DAFC and AFC elements in CFRP laminates}

The insertion and interlacing integration techniques were used to manufacture CFRP laminates with DAFC (DAFCCFRP). DAFC were integrated at three different positions; center, off-center and far off-center, in relation to the midplane of the laminate, as displayed in Fig. 2.2. In Fig 2.2 the light layers correspond to $0^{\circ}$ plies while the dark $90^{\circ}$ plies, the inclusion position is easily identified in between the continuous layers. The resin rich transition areas are indicated by the triangular space between the ply and inclusion regions. The CFRP laminates with AFC (AFC-CFRP) were prepared with the far off-center position using insertion (Fig. 2.2c) and interlacing (Fig. 2.2f) methods. Each laminate set consisted of five specimens. Integration of AFC required attention to electrical insulation of the AFC from the conductive CFRP laminate. The AFC and attached wires were laminated with Kapton ${ }^{\circledR}$ tape to ensure that no conduction paths could exist between the AFC and the conductive carbon fibers of the laminates. The wires extending from the AFC were protected by silicon tubing at the end of the specimen, and a small recess in the frame ensured wires did not move during processing. In addition, wax was applied to the wires at the recess point to protect them from encasement in resin flow during processing. 


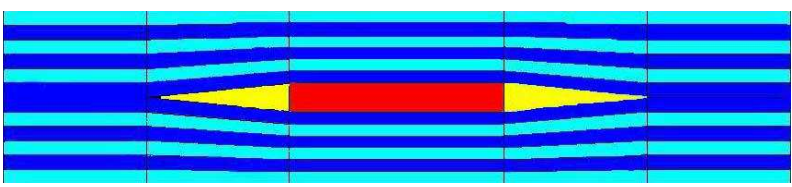

(a) Center insertion.

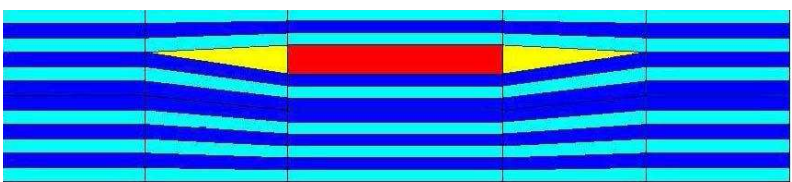

(b) Off-center insertion

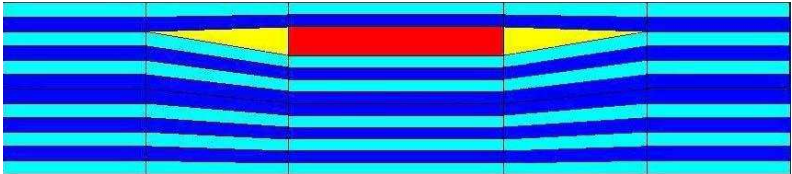

(c) Far off-center insertion

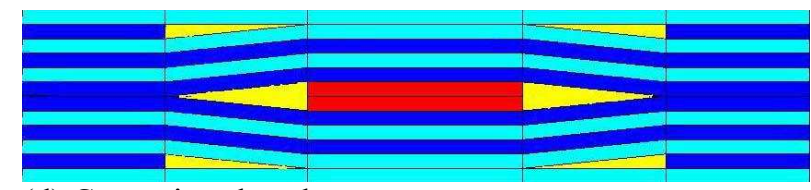

(d) Center interlaced.

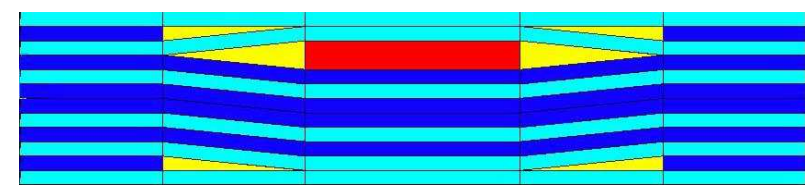

(e) Off-center interlaced

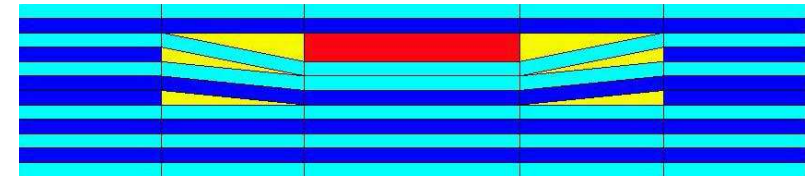

(f) Far off-center interlaced

Figure 2.2 (a-f) Summary of integrated laminate configurations for the inserted and interlaced $[0 ; 90]_{3 \mathrm{~S}}$ laminates.

\subsection{Active fiber composite and dummies manufacture}

AFC with dimensions of $33 \mathrm{~mm} \times 40 \mathrm{~mm}$ were produced for integration into the CFRP laminates. The AFC were manufactured by hand using $250 \mu \mathrm{m}$ diameter piezoelectric fibers and epoxy resin. The fibers were arranged in parallel on a grooved surface and secured with tape. The fiber layer was then transferred to an aluminum tool plate with a Kapton $^{\circledR}$ film with screen printed IDE. The epoxy was prepared with a ratio mass composition of 3:1 epoxy to hardener. Epoxy was applied to the fiber layer and the tool plate was degassed at $60^{\circ} \mathrm{C}$ to remove air from the matrix. Wires were connected to the electrodes using a conductive silver epoxy. A second Kapton ${ }^{\circledR} /$ IDE layer was laid on top and a pressure was applied for 1 hour at $120^{\circ} \mathrm{C}$. The AFC were post cured for 8 hours at $160^{\circ} \mathrm{C}$ to ensure full cure. AFC were poled at a temperature of $80^{\circ} \mathrm{C}$ for 20 minutes in air with an applied voltage of $2.5 \mathrm{kV}$. The DAFC (40mm $\mathrm{x}$ $40 \mathrm{~mm}$ ) were cut from a laminate of UD GFRP and Kapton ${ }^{\circledR}$. Two UD GFRP plies were arranged at a $+/-20^{\circ}$ orientation to one another to effectively match the longitudinal modulus of the AFC. This GFRP core was sandwiched between sheets of Kapton ${ }^{\circledR}$ film and the DAFC laminate was processed in a press at 5 bar of pressure with a temperature of $120^{\circ} \mathrm{C}$ and maintained for 90 minutes in accordance with the recommended curing cycle [22].

\subsection{Tensile Testing}

Tensile testing was performed with an Instron 1251 test machine with a $200 \mathrm{kN}$ load cell and an extensiometer $(50 \mathrm{~mm}$ gage length) was used to determine strain during the test. Both the reference laminate (containing no AFC/DAFC) and integrated (containing AFC/DAFC) specimens were prepared in accordance with ASTM Standard D 3039/D 3039M-00. Five specimens of each configuration were tested for each combination of integration procedure (insertion and interlaced) and placement position (center, off-center, far off-center). The performance of the integrated AFC (IntAFC) was monitored during tensile testing by tracing the signal produced by the AFC. A capacitor with a capacity of $1277 \mathrm{nF}$ was connected in parallel to the AFC during the test. The signal resulting from the AFC and strain data were collected via a data acquisition PC card and LabVIEW software. The signal versus applied strain behavior could then be identified and contrasted to the stress versus strain behavior of the laminate. 


\subsection{Finite element model}

A finite element model was developed in ANSYS to investigate failure in the CFRP laminate. The model of the laminate specimens with the integrated AFC/DAFC was created using the layered SHELL99 element to simulate the specimen under tensile conditions. This allowed efficient modeling of dropping layers. The regions around the inclusion area were modeled as tapered layers with material properties assigned as epoxy resin. Load levels at the point of First Ply Failure (FPF) were taken from the experimental results as an indication of failure and used to compute the numerical simulation. Tsai-Wu Strength Index (TWSI) was computed element-wise out of the resulting stress distribution corresponding to the point of FPF and plotted as thermal body load for each layer of the model.

\section{RESULTS \& DISCUSSION}

\subsection{Influence of integrated DAFC on laminate tensile properties}

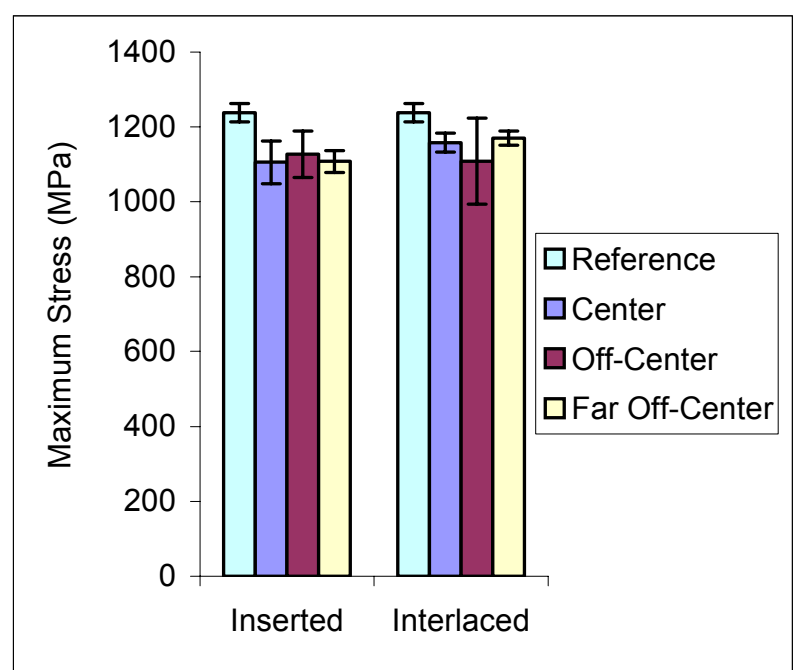

Figure 3.1 Maximum stress of the DAFC-CFRP laminates.

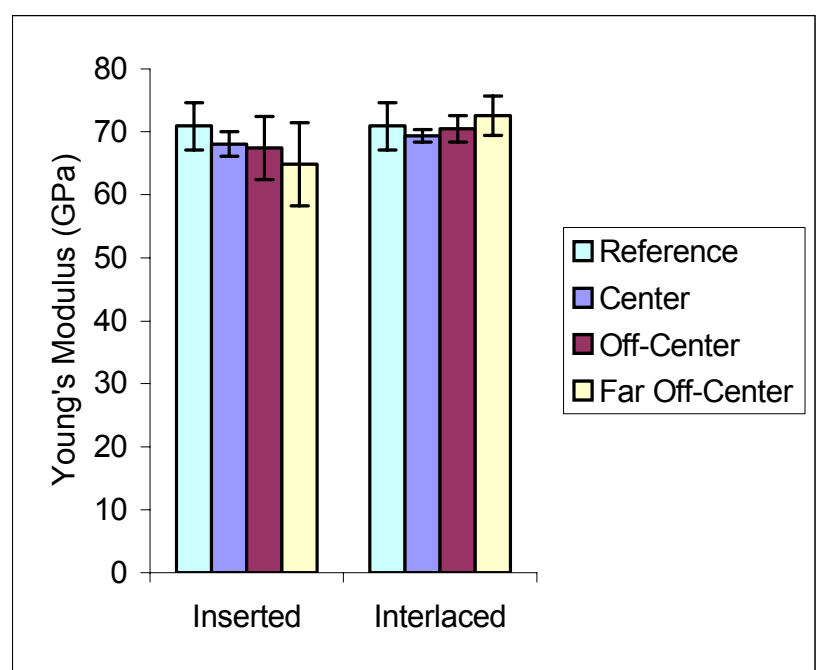

Figure 3.2 Young's modulus of the DAFC-CFRP laminates.

The results of tensile testing with the DAFC-CFRP laminates are shown in Fig 3.1 and 3.2. The affect of DAFC integration on the maximum stress value of the laminates (Fig 3.1) revealed that while there was an affect due to inclusion of the DAFC elements, the placement through the laminate thickness did not have a pronounced affect. The center, off-center, and far off-center laminates all retained similar strength values. The lack of affect was probably due to the fact that the $0^{\circ} \mathrm{CFRP}$ plies remained intact during the integration procedure. The only plies that were affected during integration were the $90^{\circ}$ plies, which were cut to allow interlacing of the laminate plies. However, the $90^{\circ}$ plies are largely non-load bearing in this tensile configuration, with the $0^{\circ}$ plies carrying most of the tensile load. The insertion and interlacing integration methods had a similar affect on the mechanical integrity of the CFRP laminates. The integration of DAFC into the CFRP laminates had a similar affect on the Young's Modulus. In the case of insertion integration the average modulus value decreased slightly as shown in Fig 3.2, however this drop is accounted for when the deviation is considered as shown in Fig 3.2. The interlaced data showed little change, and possibly an increase in stiffness as the DAFC moved away from the laminate mid-plane.

The maximum stress values for the DAFC-CFRP laminates are listed and compared to the reference laminate in Table 3.1. The difference in maximum stress values of the DAFC-CFRP laminates differed from between $5.5 \%$ to $11 \%$ as compared with the maximum strength of the reference laminate. This degradation is greater than the values reported in the literature. Mall [19] reported no affect of PZT integration on the ultimate strength of CFRP laminates, and Paget 
[16] reported an ultimate strength degradation of only $4.5 \%$ for CFRP elements with integrated PZT elements. One reason for this discrepancy may lie in the laminate configurations tested in this study. Paget [16] used a $\left[0_{4} / 90_{4} / 0_{4} / 90_{4} / 0_{2}\{\mathrm{PZT}\}\right]_{\mathrm{S}}$ laminate while the current study considers a $[0 ; 90]_{3 \mathrm{~S}}$ configuration. The ratio of the volume of the integrated element to the volume of the load bearing laminate material in this study is higher than in [16], and therefore the effect of element integration on the mechanical integrity of the laminate will be increased. The study by Mall [19] employed CFRP laminates with $[0 / \pm 45 / 90]_{S}$ and $[0 / 0 / \pm 45 / 0 / 0 / 90]_{S}$ configurations, which contain a greater number of load bearing plies relative to the number of non-load bearing plies in the laminate as compared with the current study. Therefore, the effect of integration on maximum laminate strength would be reduced as compared with the configuration considered here, which considers a laminate with an equal number of load bearing $\left(0^{\circ}\right)$ and non-load bearing $\left(90^{\circ}\right)$ plies.

Table 3.1 Summary of maximum stress values for the DAFC-CFRP laminates.

\begin{tabular}{|ccccccc|}
\hline & \multicolumn{3}{c}{ Inserted } & \multicolumn{3}{c|}{ Interlaced } \\
Center & Off Center & Far Off-Center & Center & Off Center & Far Off-Center \\
Avg. (MPa) & 1105 & 1127 & 1107 & 1158 & 1108 & 1169 \\
Std. Dev. & 57 & 62 & 29 & 25 & 115 & 52 \\
Std. Dev. (\%) & 5.2 & 5.5 & 2.6 & 2.2 & 10.4 & 4.5 \\
Ref. Diff. (\%) & 11.0 & 5.5 & 10.5 & 6.5 & 10.5 & 5.5 \\
\hline
\end{tabular}

\subsection{Performance of IntAFC Laminates}

The affect of far off-center AFC integration on the CFRP laminates is displayed below in Fig 3.3 and 3.4. Similar to the DAFC-CFRP findings, the Young's Modulus of the laminates with AFC stayed constant with respect to the modulus of the reference laminate. The maximum stress at break decreased with both insertion and interlacing integration methods, but between insertion and interlacing the values are nearly identical. The difference between the two integration techniques with AFC-CFRP laminates is very similar to the DAFC-CFRP results.

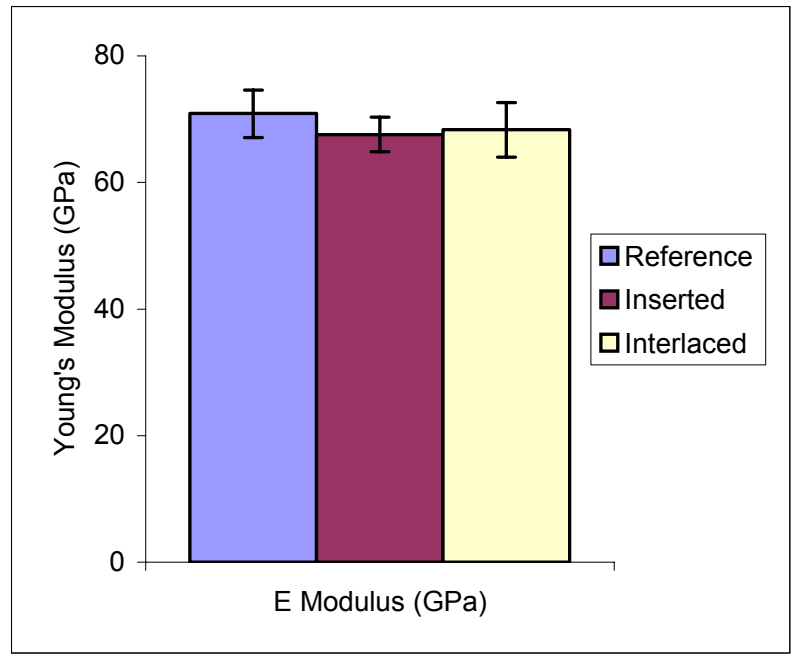

Figure 3.3 Young's Modulus of AFC-CFRP laminates.

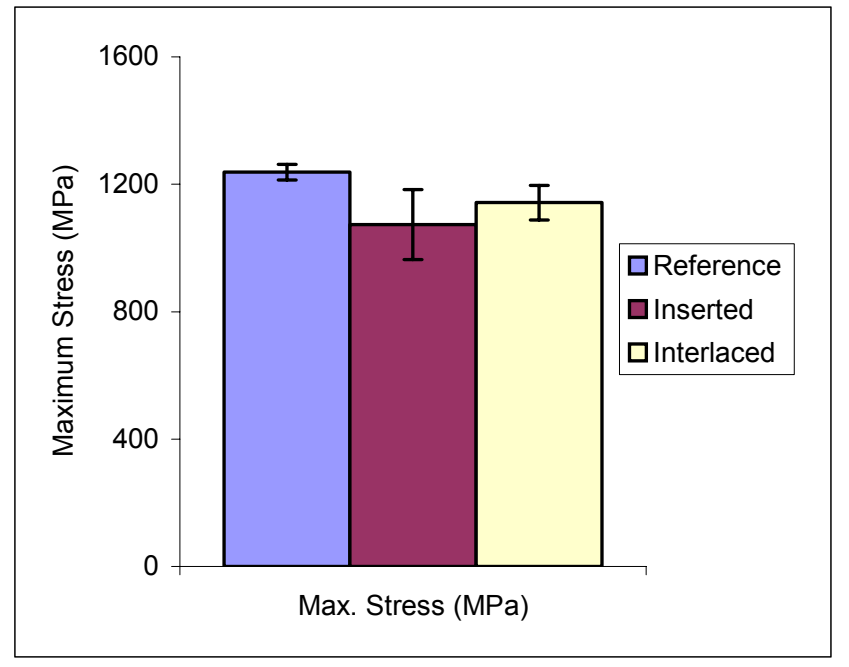

Figure 3.4 Maximum stress of AFC-CFRP laminates.

The maximum stress values for the AFC-CFRP laminates are summarized in Table 3.2. As compared with the DAFCCFRP laminates the AFC had a slightly greater affect on the ultimate strength of the laminate. The far off-center inserted AFC configuration degraded the laminate strength by $13.3 \%$ while the DAFC configuration only showed a $10.5 \%$ reduction in strength. The far off-center interlaced AFC configuration degraded the laminate strength by $7.8 \%$, while the DAFC-CFRP laminate showed a $5.5 \%$ reduction. These relative differences may be due to the failure character of the DAFC as compared with the AFC. 
Table 3.2 Summary of maximum stress values for the AFC-CFRP laminates.

\begin{tabular}{|ccc|}
\hline & Inserted & Interlaced \\
Avg. (MPa) & 1073 & 1142 \\
Std. Dev. & 109 & 54 \\
Std. Dev. (\%) & 10.2 & 4.8 \\
Ref. Diff. (\%) & 13.3 & 7.8 \\
\hline
\end{tabular}

The electromechanical performance of the IntAFC was assessed by tracking the voltage signal from the AFC during testing. The data for both insertion and interlaced AFC-CFRP laminates is shown in Fig 3.5. During tensile testing most of the integrated AFC specimens displayed near-linear voltage signal to strain behavior up to about 0.005 strain. Beyond 0.005 strain the signal became increasingly nonlinear and saturated between 0.01 and 0.015 , taking on a plateau character until final failure in the laminate. This negative slope could be due to discharge of the capacitor. The stress/strain curves for the AFC-CFRP laminates are depicted in Fig. 3.6, where the FPF points are noted. The FPF corresponds to the first "kink" or inconsistency in the stress/strain behavior, which is taken as evidence of failure in the laminate. The defined FPF values were then used to compute the Tsai-Wu failure prediction plots.

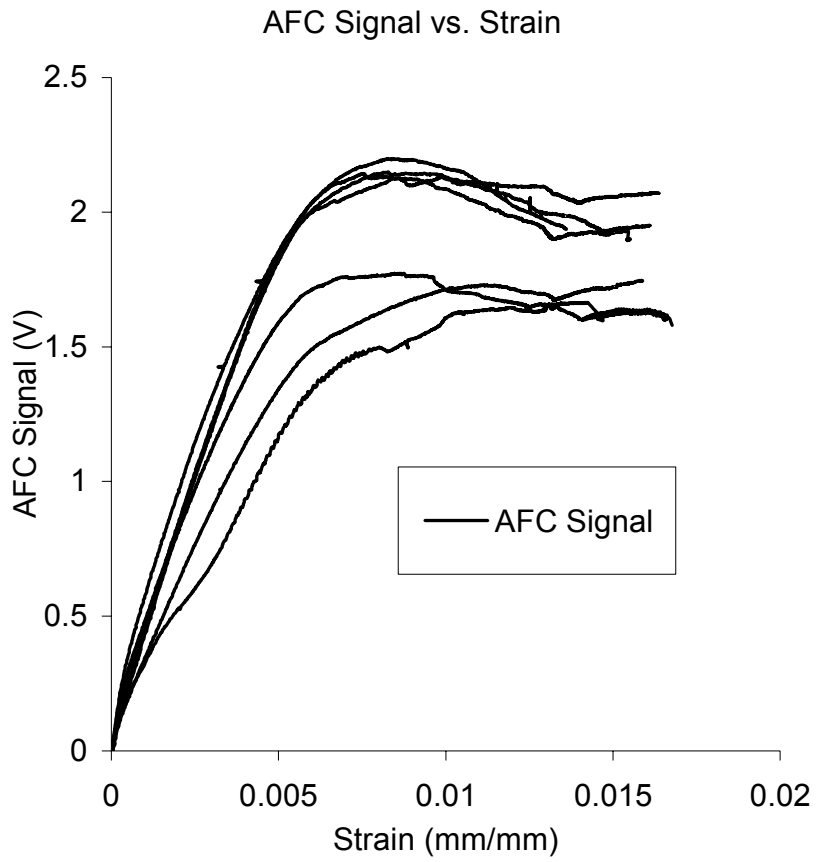

Figure 3.5 AFC signal in response to tensile strain.
IntAFC Stress vs. Strain

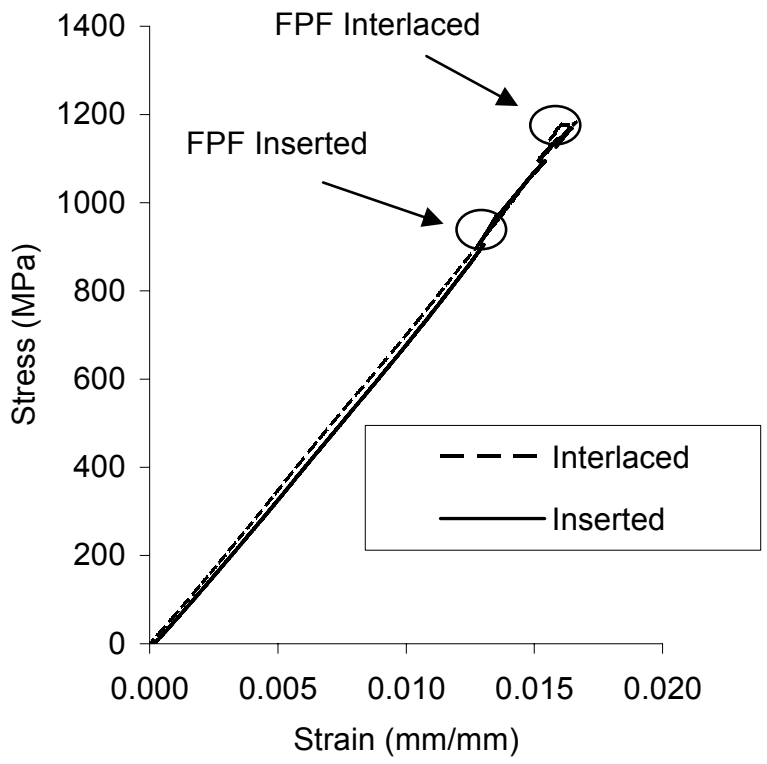

Figure 3.6 Stress/strain plots for AFC-CFRP laminates.

\subsection{Laminate Fracture Behavior}

In Fig. 3.7 both the AFC-CFRP insertion in (Fig. 3.7a) and interlacing (Fig. 3.7b) specimens post tensile testing are shown. Fracture patterns in the laminates were uniform across the different integration configurations and occurred in the region of the AFC. As can be clearly seen, transverse fracture through the width of the tensile specimens occurred at the top and bottom edge of the inclusion integration region. The transverse cracks are brittle in nature and run perpendicular to the load direction, situated in the vicinity of the AFC top and bottom edges. The fracture in the inserted laminate is more localized as compared with the interlaced specimen. Fig. 3.7b shows more extensive delamination and failure on the surface of the interlaced laminate. 

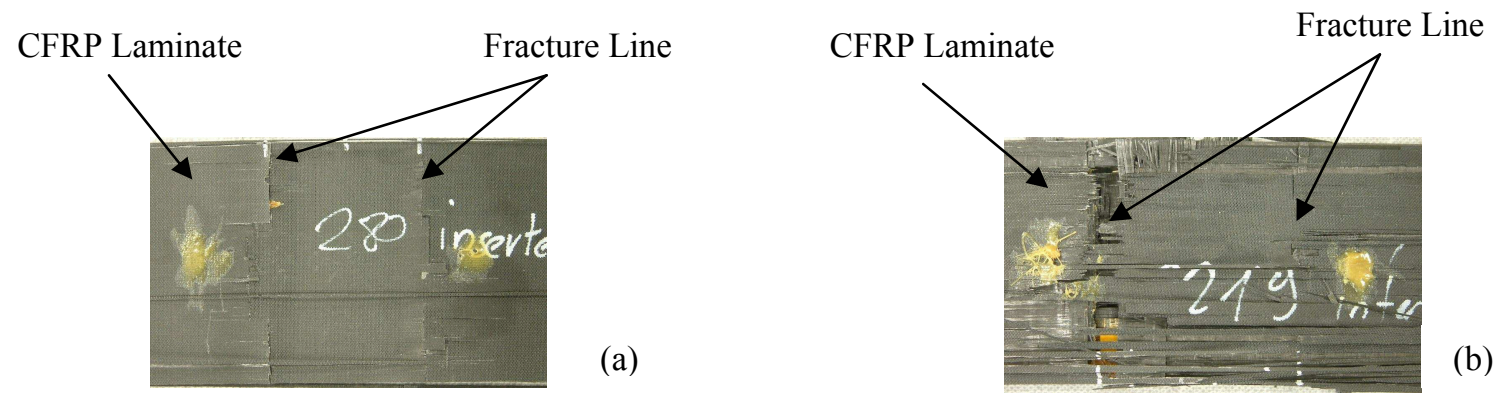

Figure 3.7 Fracture behavior of AFC-CFRP laminates using the insertion (a) and interlacing (b) integration techniques.

The forces corresponding to the FPF points indicated in Fig. 3.6 were used to compute the Tsai-Wu failure criterion for the ANSYS model. The FPF points in the stress-strain curved implied damage initiation in the laminate, and therefore the Tsai-Wu criterion was calculated for these corresponding forces. Tsai-Wu was calculated at the FPF points since performing the calculation at the exact point of ultimate failure would only confirm that failure should have occurred at that point. The goal of implementing the Tsai-Wu criterion was to begin establishing a reference for how damage is initiating in the laminates in relation to the integrated AFC. A Tsai-Wu calculation at or above a value of 1 indicates failure in the element. Fig. 3.8 shows the Tsai-Wu failure criterion in the top ply of the laminate for each element in the model for the far off-center insertion (Fig. 3.8a) and interlacing (Fig. 3.8b) configurations. The Tsai-Wu plot predicted failure at the inclusion edge, showing an indication of damage initiation. The insertion plot (Fig 3.8a) indicates failure at the corner edges of the inclusion region while the interlacing plot (Fig 3.8b) predicts failure directly at the edge of the inclusion region. A comparison between the Tsai-Wu plots and the fracture evidence from the tensile specimens suggests good correlation between the experimental and numerical findings. Even though the AFC are integrated into $90^{\circ}$ non-load bearing plies, there is still an affect on the damage and failure characteristics of the laminates due to their presence.
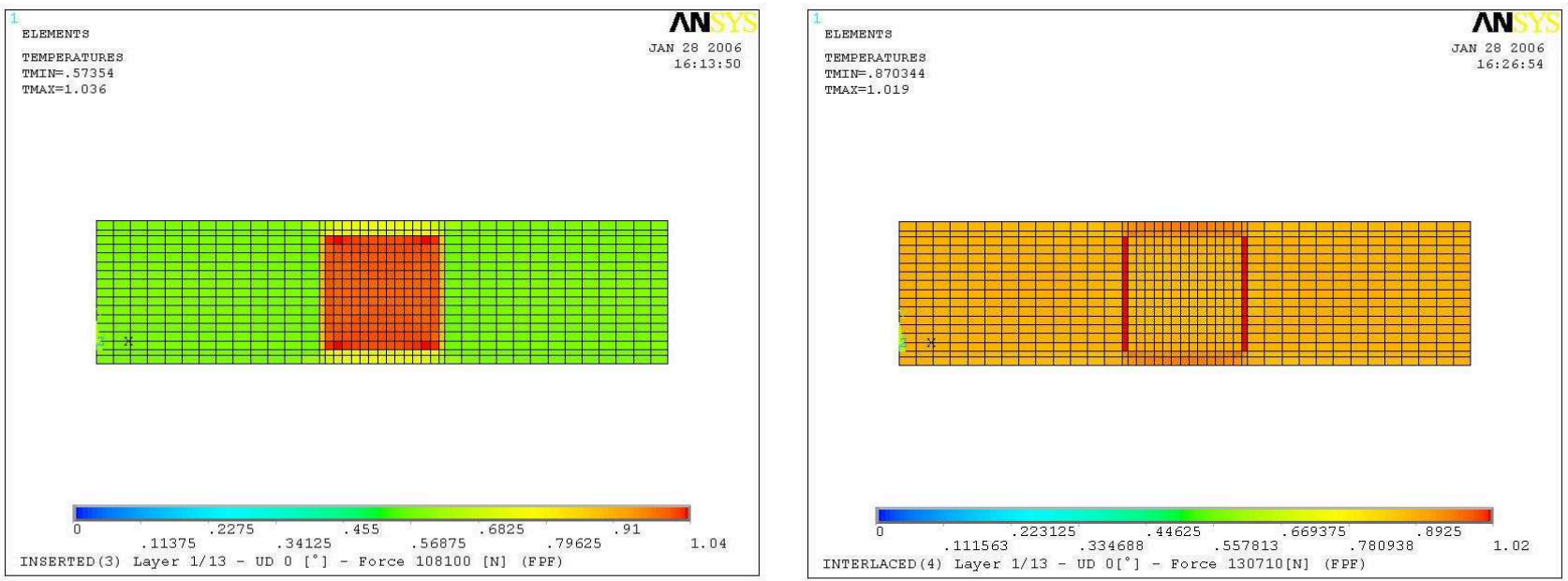

(a)

(b)

Figure 3.8 Tsai-Wu plots of the first laminate ply from the ANSYS model with insertion (a) and interlacing (b) integration.

The difference between insertion and interlacing is evident in the distribution of Tsai-Wu values. In Fig. 3.8 the insertion model shows concentrated failure prediction at the corners of the integration region, but very high Tsai-Wu 
values are also seen over the entire integration region. With the insertion configuration, the integration region severs as failure concentration point. By contrast, the interlacing technique seems to better distribute the critical forces over the entire laminate. However, the edge of the integration region remains a critical point of failure. This is also supported by the failure character of the experimental specimens. While the inserted specimen in Fig. 3.7a shows primarily transverse cracking, the interlaced specimen (Fig. 3.7b) also shows extensive failure of the surface ply.

The Tsai-Wu plots for the second ply layer $\left(90^{\circ}\right.$ non-load bearing) are depicted in Figure 3.9 for both insertion (a) and interlacing (b) integration. Here the distribution in the inserted configuration shows a diamond-like pattern over the integration region. Since this is a non-load bearing ply it is expected for the failure index to be rather low. While the TWSI is not critical in this case, it does show that the edge of the integration region is still seeing the greatest affect due to loading. Characterizing the transfer of critical forces through the laminate could be used as a design constraint when considering the type of geometry to use for engineering a sensor for a smart materials application in this type of loading environment. In the second layer of the interlaced laminate (Fig. 3.9b) the edges of the integration region are also seeing the highest TWSI value. While the region as a whole is not prone to failure it shows that the forces are not moving through the integration region in the interlaced case.
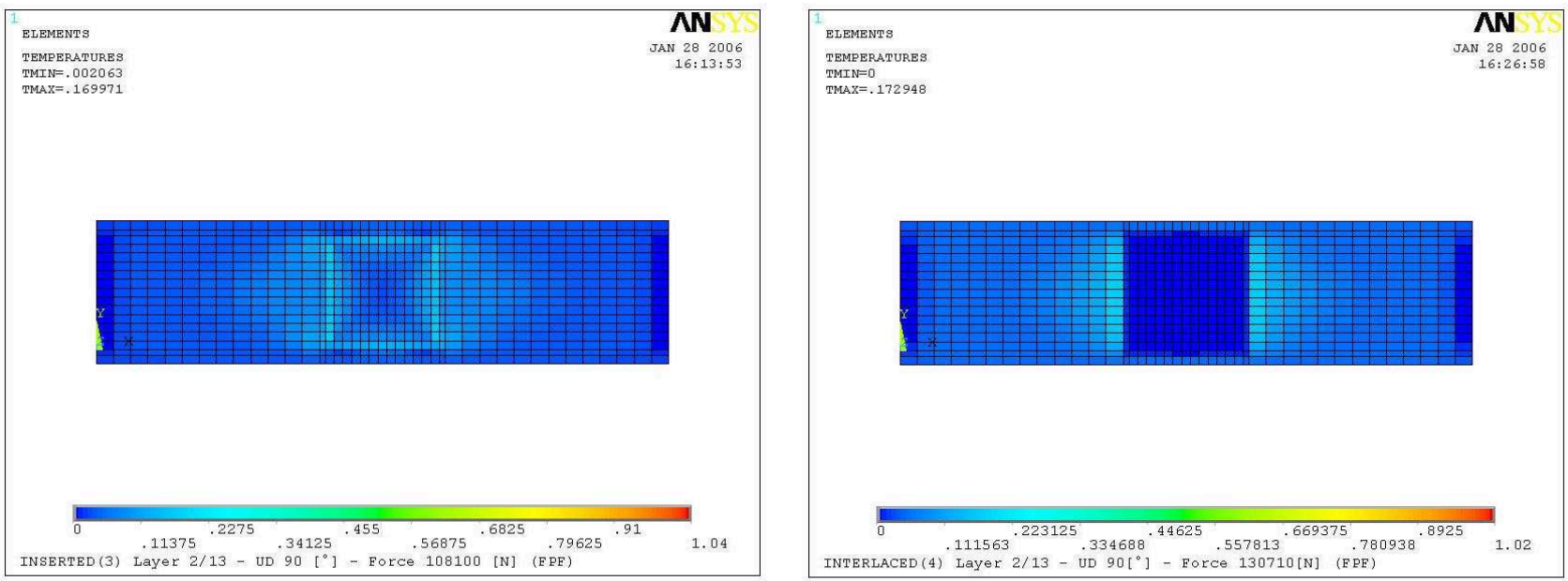

(a)

(b)

Figure 3.9 Tsai-Wu plot of the second laminate ply from the ANSYS model with insertion (a) and interlacing (b) integration.

The Tsai-Wu plots for the third ply layer $\left(0^{\circ}\right.$ load bearing) are depicted in Figure 3.10 for both insertion (a) and interlacing (b) integration. Here it is interesting to note that the only area with a TWSI grater than 0 is the integration region. This implies that the forces are still being transferred through that region. The interlaced configuration again shows a high TWSI distribution over the entire model, with a critical value of 1 at the edge of the integration region. From this standpoint, it appears as though the insertion method might be more detrimental to the performance of an integrated smart element since the force is being transferred primarily through the integration region. 

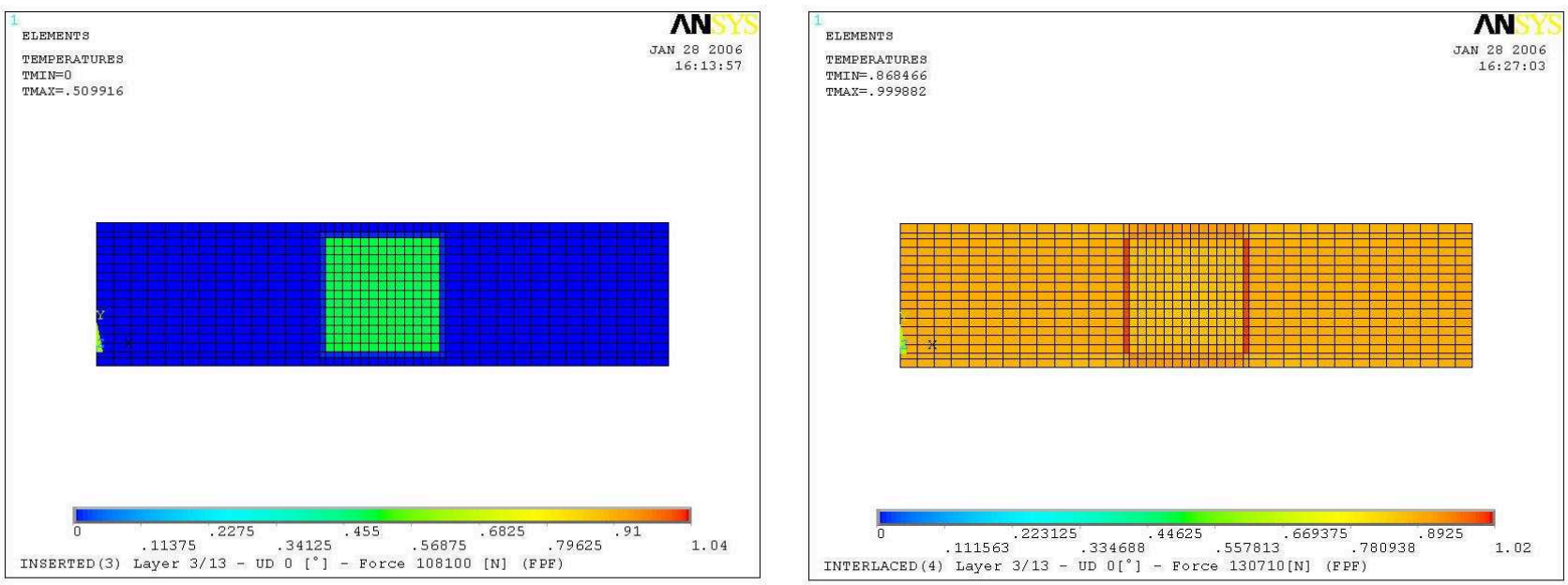

(a)

(b)

Figure 3.10 Tsai-Wu plot of the third laminate ply from the ANSYS model with insertion (a) and interlacing (b) integration.

\section{CONCLUSION}

The current work investigated the affect of AFC integration on the mechanical integrity of CFRP laminates. While integrating $\mathrm{DAFC} / \mathrm{AFC}$ in $[0 ; 90]_{3 \mathrm{~S}}$ laminates did degrade the mechanical properties of those laminates (as compared with the reference laminate), varying the placement through the laminate thickness did not further degrade laminate integrity. The reduction in ultimate strength of the laminates in the current study is greater than in the literature, but this is attributed to the use of different laminate configurations. Using the TWSI, damage initiation was predicted at the edge of the AFC/DAFC integration region. This location correlated with the fracture patterns observed in the laminates after tensile testing. The TWSI plots of the laminates showed that forces were transferred through the integration region in the inserted case. With interlacing the critical forces were distributed over the load bearing plies, and the critical point of failure was predicted to be at the interface edge between the integration region and the surrounding ply. Implementing failure criterion such as Tsai-Wu adds another window to investigate and eventually optimize the integration procedure for $\mathrm{AFC}$ or similar planar-like actuators and sensors.

\section{ACKNOWLEDGMENTS}

The authors would like to acknowledge the contribution of Alain Monnin in the development of this work. Funding for this work was provided by ETH Zurich and by EMPA.

\section{REFERENCES}

1. Haertling GH "RAINBOW Ceramics - A new type of ultra-high-displacement actuator" Amer. Ceram. Soc. Bull. 73 93-96, 1994

2. Mossi KM, Selby GV, Bryant RG "Thin-layer composite unimorph ferroelectric driver and sensor properties" Mater. Lett. 35 39-49, 1998

3. Bent AA, Hagood NW "Piezoelectric fiber composites with interdigitated electrodes" J. Int. Mat. Syst. Struct. 8 903-919, 1997

4. Lynch CS "The effect of uniaxial stress on the electro-mechanical response of 8/65/35 PLZT" Acta Mater. 44 41374148, 1996

5. Cao H, Evans AG "Nonlinear deformation of ferroelectric ceramics" J. Am. Ceram. Soc. 76 890-896, 1993 
6. Tanimoto T, Yamamoto K, Morii T "Nonlinear stress-strain behavior of piezoelectric ceramics under tensile loading" Proc. 9th IEEE International Symp. on Applications of Ferroelectrics, 1995

7. Schäufele AB, Härdtl KH "Ferroelastic properties of lead zirconate titanate ceramics" J. Am. Ceram. Soc. 79 26372640, 1996

8. Wickramasinghe VK, Hagood NW "Materials characterization of active fiber composites for integral twist-actuated rotor blade application" Smart. Mater. Struct. 13 1155-1165, 2004

9. Head inc. Active Electronic Dampening System (EDS) Protector Racquet www.head.com/tennis/

10. Head inc. IntelligenceTM Ski System www.head/ski.com

11. Hall SR, Prechtl EF "Development of a piezoelectric servoflap for helicopter rotor control" Smart Mater. Struct. 5 26-34, 1996

12. Giurgiutiu V "Review of Smart-Materials Actuation Solutions for Aeroelastic and Vibration Control" J. Intelligent Mater. Sys. and Struct. 11 525-544, 2000

13. Crawley EF, de Luis, J "Use of piezoelectric actuators as elements of intelligent structures" AIAA 25 1373-1385, 1987

14. Vizzini AJ, Xingh DA "Structural integrity of composite laminates with interlaced actuators" Smart Mater. Struct. 6 71-79, 1994

15. Vizzini AJ, Shukla DR "Interlacing for improved performance of laminates with embedded devices" Smart Mater. Struct. 5 225-229, 1996

16. Paget CA, Levin K "Structural integrity of composites with embedded piezoelectric ceramic transducer" SPIE Proc. 3668 306-313, 1996

17. Mall S, Coleman JM "Monotonic and fatigue loading behavior of quasi-isotropic graphite/epoxy laminate embeded with piezoelectric" sensor Smart Mater. Struct. 7 822-832, 1998

18. Mall S, Hsu TL "Electromechanical fatigue behavior of graphite/epoxy laminate embedded with piezoelectric actuator" Smart Mater. Struct. 9 78-84, 2000

19. Mall S "Integrity of graphite/epoxy laminate embedded with piezoelectric sensor/actuator under monotonic and fatigue loads" Smart Mater. Struct. 11 527-533, 2000

20. Yocum M, Abramovich G, Grunwald A, Mall S "Fully reversed electromechanical fatigue behavior of composite laminate with embedded piezoelectric actuator/sensor" Smart Mater. Struct. 12 556-564, 2003

21. Melnykowycz M, Kornmann X, Huber C, Brunner AJ, Barbezat M 2005 Integration of Active Fiber Composite (AFC) Sensors/Actuators into Glass/Epoxy Laminates SPIE International Symposia Smart Structures \& Materials/NDE March 7-10th 2005, San Diego, CA

22. Stesatape EP121-GR101/228-30 Product Data Sheet. Stesalit Inc, www.stesalit.com. 